\title{
Wege aus der Krise bei psychischer Krankheit
}

\author{
Mit einem Integrationscoaching zurück in die Wirtschaft
}

Gute Fallbeispiele zeigen immer wieder, dass es Wege aus einer psychischen Krise gibt. Dazu ist eine nahtlose Vernetzung in der psychosozialen Rehabilitation ohne lange Wartezeiten und Übergänge für Betroffene Voraussetzung. Ziel ist, die größtmögliche Lebensqualität und Selbstständigkeit im Alltag wieder zu erlangen.

Maria, 44 Jahre, kam auf Zuweisung des Betreuungsheimes Tillmitsch im Jänner 2004 zur Arbeitsrehabilitation in den werkdienst-süd, eine Trainingsfirma zur beruflichen Integration für psychisch beeinträchtigte Menschen in der Struktur eines Kleinbetriebes mit unterschiedlichen Dienstleistungsangeboten. Diagnose: chronische paranoide Schizophrenie

Bis 1998 war Maria wohnhaft im eigenen Haus in Graz, verheiratet, sie hat zwei Kinder. Es kam zum ersten stationären Aufenthalt in der Landesnervenklinik Sigmund Freud, Graz, mit der Diagnose paranoide Schizophrenie. Es folgten Arbeitsplatzverlust (Sekretärin), Scheidung und nach mehreren stationären Aufenthalten die vollstationäre Aufnahme in das Betreuungsheim Tillmitsch. Maria bekam auch einen Sachwalter.

Als Maria die Arbeitsrehabilitation im werkdienst-süd begann, war sie noch schwer hospitalisiert. Sie hatte zwar im Wohnheim bereits größere Freiheiten als andere Bewohner, weil sie als vereinbarungsfähig und compliant galt, sie war es aber nicht gewohnt, ihren Alltag selbst zu planen und zu gestalten. Anfangs mussten alle Arbeitsschritte im Arbeitstraining in sehr kleine Einheiten unterteilt und vorgegeben werden. Maria verfügte nur über ein Mindestmaß an Selbstvertrauen und hatte keine wirklich konkreten Zukunftsvorstellungen.

Durch die Zusammenarbeit mit anderen Teilnehmern lernte sie, sich selbst mit ihren Fähigkeiten einzuschätzen und wurde auch neugierig auf für sie bis dato neue Betätigungsfelder. Innerhalb des psychoedukativen Gruppenangebotes erfuhr Maria viel Informatives über ihr Krankheitsbild und auch mögliche Bewältigungstechniken. Erstmals verdiente sie auch wieder ihr eigenes Geld, da sie im

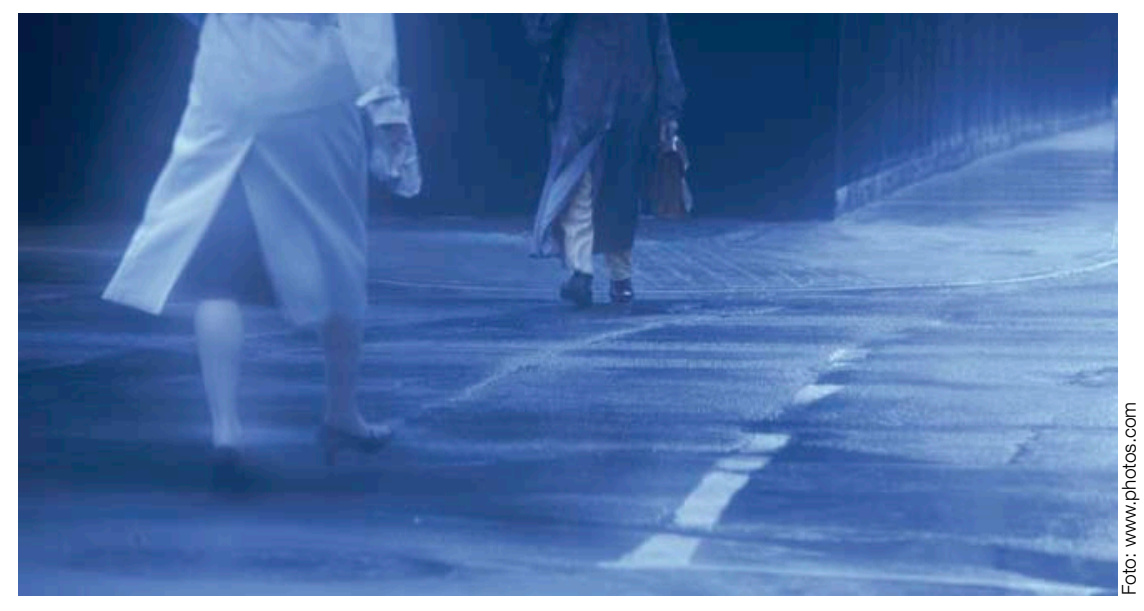

Nach psychischer Krankheit ist der Schritt zurück ins Berufsleben nicht leicht, aber es gibt Hilfe.

Rahmen der Arbeitsrehabilitation über das Bundessozialamt im werkdienst-süd angestellt wurde. Sie hatte aber aufgrund der Besachwalterung und der Heimunterbringung nicht wirklich freien Zugang zu ihrem Gehalt. In einer Helferkonferenz zwischen Sozialarbeitern und Pflegedienstleitung des Betreuungsheimes und den Betreuern von werkdienst-süd wurden Möglichkeiten diskutiert, wie Maria wieder zur selbstständigen Lebensführung gelangen kann. Als erster Schritt wurde die Sachwalterschaft aufgehoben, da Maria inzwischen aus unserer Sicht durchaus fähig war, sich selbst wieder um ihre finanziellen und behördlichen Belange zu kümmern. Marias Haus in Graz war in der Zwischenzeit ziemlich heruntergekommen. Der Garten war verwildert und es musste auch im Haus einiges saniert werden. Im Rahmen der Arbeitsgruppen des werkdienst-süd wurden ihr Garten und ihr Haus mit ihr gemeinsam „auf Vordermann“ gebracht. Vom Heim erhielt sie die Möglichkeit, am Wochenende tageweise dorthin zurückzukehren. Ein Sozialarbeiter begleitete sie zunächst bei der Hinfahrt und bei den Einkäufen. Nach einigen Wochen konnte sie bereits selbstständig, mit öffentlichen Verkehrsmitteln wieder dorthin fahren und ihren Haushalt am Wochenende führen.

Ihre inzwischen erwachsenen Kinder nahmen wieder verstärkt Kontakt zu ihr auf, sie erlebten ihre Mutter wieder als nahezu selbstständige Person. Im werkdienst-süd hatte sich Maria im Laufe eines Jahres zu einer Stütze des Arbeitsteams der Küche und Hauswirtschaft gemacht. Sie arbeitete überaus genau und war flexibel in der Auftragsabwicklung geworden. Maria kehrte 2005 ganz in ihr Haus nach Graz zurück und kam mit dem Zug täglich in den werkdienst-süd nach Lebring. Sie war psychisch stabil und hoch dosierte Neuroleptika wurden nach zweijähriger Symptomfreiheit ausgeschlichen, eine geringe Erhaltungsdosis blieb. Nun wurde ein Arbeitsplatz in der freien Wirtschaft mit ihr im Rahmen des Integrations-Ccoachings gesucht. Maria bekam eine Stelle als Reinigungskraft in einer Rechtsanwaltskanzlei in Graz. Ihr Stundenkontingent wurde nach einem halben Jahr auf 75 Prozent aufgestockt und sie wurde zur Leiterin der Reinigungsabteilung dieser Firma. Sie ist nun verantwortlich für den Einkauf und die Verwaltung aller Reinigungsmaterialien und hat zwei Kolleginnen. Nebenbei konnte sie bereits auch Schreibarbeiten (da sie im PC-Training im werkdienst-süd neue Computerkenntnisse gelernt hatte) in der Kanzlei durchführen. Maria hält bis heute brieflichen Kontakt mit dem werkdienst-süd und ist weiterhin psychisch stabil.

\section{Korrespondenz:}

Mag. Helene Prack

PSD-Leibnitz

TS-Klapotetz, Sailergasse 8, 8430 Leibnitz

E-Mail: helene.prack@gfsg.at 